\title{
SEMIOLOGIA NEUROLÓGICA NUMA POPULAÇÃO DE CRIANÇAS DEFICIENTES AUDITIVAS
}

\author{
VANDA M. GIMENES GONCALVES *, ANA MARIA S. G. PIOVESANA ** \\ MARIA VALERIANA L. DE MOURA-RIBEIRO ***
}

\begin{abstract}
RESUMO - Foi constituída uma amostra aleatória de 42 crianças deficientes auditivas neurossensoriais congênitas, profundas e bilaterais, com idade cronológica variando entre 4 e 7 anos, que frequentavam classes de habilitação da cidade de Campinas. As crianças propostas foram comparadas com dois grupos controles de 42 crianças, da mesma faixa etária, de classe comum. Todas foram submetidas ao exame neurológico tradicional. Verificou-se que os aspectos que demonstraram diferenças foram o perímetro craniano e o tono muscular. Nos demais itens aviliados, mostrou-se hiperatividade motora, sindrome cerebelar e sindrome ocular, porém não houve diferença significativa entre os dois grupos.
\end{abstract}

PALAVRAS-CHAVE: perda auditiva, sensorioneural; exame neurológico.

\begin{abstract}
Neurologic semiology in a sample of hearing impaired children.
\end{abstract}
SUMMARY - A random sample of 42 sensorioneural hearing implaired children (severe and bilateral) was studied, from special classes in Clampinas, with chronological ages varying between 4 and 7 years old. The children of this sample were compared with two control groups of 42 children of the same chronological age, from regular ciasses of private and public schools. All of them were submitted to the traditional neurological examination. Hearing impaired children showed differences as to head circumference and muscle tonus. In the other examined items we found motor hyperactivity, cerebellar and ocular syndromes although there were no significant differences between the groups.

KEY WORDS: hearing loss, sensorioneural; neurologic examination.

Poucos autores têm-se preocupado com as alterações neurológicas da criança deficiente auditiva (DA). Assim, Horak e col.7 referem que em seus pacientes a avaliação pelo neurologista infantil "não revelou sinais neurológicos indicativos de lesão cerebral localizada, embora todos pudessem ser classificados como tendo mínimas disfunções cerebrais, com base em déficits sutis de coordenação motoran. Por outro lado, Rapin 12 valoriza a semiologia neurológica da criança DA, lembrando que malformações do ouvido são frequentemente associadas com malformações de algumas estruturas de fossa posterior, notadamente agenesia nuclear. Estão melhor definidos os aspectos do desenvolvimento neuromotor da criança DA. Assim, Rapin 10 e Castagno \& Carvalhal 1 encontraram média de idade de 15 meses para a marcha independente e caracterizaram retardo neuromotor nessas crianças.

Tendo como objetivo a semiologia neurológica da criança DA, a presente pesquisa foi elaborada visando conhecer esses aspectos na população habilitada isa cidade de Campinas.

Departamento de Neurologia, Faculdade de Ciências Médicas (FCM) da Universidade Estadual de Campinas (UNICAMP); * Professor Assistente Doutor; ** Professor Assistente; *** Professor Livre-Docente. Este estudo constitui parte do trabalho apresentado na FCM/ UNICAMP, como Tese de Doutorado da autora. Aceite: 28-novembro-1992.

Dra. Vanda Maria Gimenes Gonçalves - Departamento de Neurologia, Hospital das Clínicas, UNICAMP - Caixa Postal 6111 - 13081-970 Campinas SP - Brasil. Fax 55.192.394717. 


\section{CASUISTICA L MgTODOS}

De uma população de 60 crianças DA neurossensoriais congênitas, foram sorteadas 42 , com idade cronológica variando de 4 a 7 anos e 11 meses. Essas crianças frequentavam 5 classes de habilitaçăo da cidade de Campinas. A distribuição foi de 8 crianças com 4 anos, 13 com 5 anos, 11 de 6 anos e haviam 10 crianças com 7 anos.

Para diagnosticar e quantificar a deficiência auditiva, todas elas tinham eletrococleografia, realizada em diferentes aparelhos. A severidade da perda auditiva variou entre moderada $(45$ a $69 \mathrm{db})$, severa $(65$ a $80 \mathrm{db})$ e profunda $(>85 \mathrm{db})$.

Foram comparadas a dois grupos controles, num total de 84 crianças, que apresentavan produçấo escolar normal em classe comium de escola particular e da rede oficial de ensino. selecionadas entre crianças da mesma faixa etária.

Todas foram submetidas ao Exame Neurológico Tradicional (ENT) padronizado por Lefèvre 8. As medidas de crânio foram obtidas seguindo a sistematização proposta por Diament 3. Calculou-se o indice cefálico novo (ICn) determinado por Diament \& Rodrigues 4.

Os dados foram processados em computador da linha Scopus, Nexus 2600, 16 bits, por meio do pacote computacional estatístico SAS (Statistical Analysis System). Os testes $\chi^{2}$ de Pearson foram utilizados, para determinar correlações estatisticamente significantes. $O$ nivel de significancia adotado foi de $5 \%$.

\section{HESTLTADOS}

O ENT foi normal em 42 controles $(n=84)$ e detectou deficiéncia auditiva pura em 11 crianças $(n=42)$ (Tabela 1).

Na avaliação do perímetro craniano (Tabela 2), comparando-se os dois grupos, verificou-se que as crianças deficientes auditivas apresentaram média de perímetro craniano significativamente menor em todas as faixas etárias $(p=0,001)$, excluidos os casos de microcefalia, observada em 4 deficientes auditivos.

Tabela 1. Alterações neurológicas observadas nas crianças controles e nas deficientes auditivas.

$\begin{array}{ccc} & \text { Crianças } & \text { Deficientes } \\ \text { Exame neurológico } & \text { controles } & \text { auditivas } \\ & (n=84) & (n=42)\end{array}$

Sem alterações

Deficiência auditiva pura

Microcefalia

Hiperatividade

Hipotonia muscular

Sindrome cerebelar

axial

apendicular

Sindrome ocular

estrabismo

hiperpigmentação

nistagmo congênito
42

0

0

0

40

0

2

0
0

11

4

4

26 
o indice cefálico novo (ICn) apresentou valor minimo de 0,88 e máximo de 1,007 , valor médio e mediana de $\mathbf{0 , 9 6}$. Não houve diferença significativa no $\mathrm{ICn}$, quando considerados os grupos $(p=0,18)$, sexo $(p=0,38)$ e idade cronológica $(p=0,40)$.

Na avaliação do tono muscular, encontrou-se hipotonia de membros em 40 crianças controles e 26 deficientes auditivas. Considerando-se sua distribuição em relação à faixa etária (Tabela 3) verificou-se que nos DA, com o aumento da idade, houve aumento no número de crianças apresentando hipotonia muscular. Porém, esse resultado não pode ser considerado significativo, pelo pequeno número de crianças avaliadas em cada faixa etária.

Nos demais itens avaliados pelo ENT, não houve diferença significativa entre os grupos.

Tabela 2. Médias dos perímetros cranianos, em $\mathrm{cm}$, das crianças controles e deficientes auditivas, segundo a idade cronológica.

\begin{tabular}{|c|c|c|c|c|}
\hline Grupos & \multicolumn{4}{|c|}{ Idade cronológica } \\
\hline Crianças controles & 51,83 & 51,25 & 52,47 & 52,05 \\
\hline d. p. & 1,17 & 1,41 & 1,83 & 1,51 \\
\hline Deficientes auditivas & 49,14 & 49,30 & 49,81 & 50,00 \\
\hline d. $p$. & 1,18 & 2,06 & 1,61 & 1,58 \\
\hline
\end{tabular}

d.p., desvio-padrão.

Tabela 3. Distribuição da hipotonia muscular segundo a idade cronológica.

\begin{tabular}{|c|c|c|c|c|c|}
\hline \multirow[b]{2}{*}{ Grupos } & \multicolumn{5}{|c|}{ Idade } \\
\hline & & 4 anos & 5 anos & 6 anos & 7 anos \\
\hline \multirow[t]{2}{*}{ Controles } & + & 8 & 12 & 11 & 9 \\
\hline & - & 8 & 14 & 11 & 11 \\
\hline \multirow[t]{2}{*}{ Def. auditivas } & + & 2 & 6 & 8 & 10 \\
\hline & - & 6 & 7 & 3 & 0 \\
\hline
\end{tabular}

+. presença de hipotonia; - ausência de hipotonia.

\section{COMENTARIOS}

Há poucos dados na literatura sobre aspectos neurológicos de populações de escolas comuns. Towen 16 chama a atenção para a grande dificuldade que existe na interpretação dos achados. Esse autor enfatiza que um sinal neurológico isolado, por exemplo dorsiflexão isolada do hálux ou exaltação do reflexo patelar, raramente tem significado clínico. Esse fato de importância fundamental. também foi mencionado por Lefèvre 9: "como saber se uma pequena anormalidade encontrada no exame neurológico à qual possamos, corretamente, atribuir significado patológico, deve ser simplesmente relacionada com um distúrbio de comportamento ou aprendizado. Torna-se necessário um aguçado espírito crítico, não apenas para distinguir o realmente patológico, do que cai dentro da variação normal».

As medidas dos perímetros cranianos das crianças controles desta pesquisa estiveram dentro dos limites propostos por Diament \& Rodrigues 4 . No entanto, há poucas referências sobre a população escolar normal. Assim, Sells 15 avaliou 
o perímetro craniano de 1006 estudantes entre 5 e 18 anos de idade e encontrou prevalência de $1,9 \%$ de microcefálicos entre crianças sem deficiência mental, frequentando classes regulares. Em geral, a microcefalia é associada com inteligência abaixo da média. Contudo, há pessoas microcefálicas que obtêm alta pontuação nos testes cognitivos $5,15,17$, demonstrando que a relação entre microcefalia e retardo mental não é tão certa como se acreditava, quando se tomou por base os estudos realizados em certas populações selecionadas.

Comparando os perímetros cranianos das crianças controles com as DA, verificou se que apesar de estarem dentro dos limites de normalidade determinados por Diament \& Rođrigues 4, esses perímetros situavam-se abaixo da média, com perímetro craniano significativamente menor nos DA.

E referido por vários autores que o tamanho da cabeça pode ser fator meramente familiar ou reflexo do tamanho da criança ou ainda, representar o extremo da variação normal 2,6,18. Assim, como foi referido por Rosado e col.13, o conhecimento amplo dos fatores que influenciam o desenvolvimento cerebral da criança deve ser constantemente revisado, procurando estabelecer compara. çōes entre diferentes populaçōes, como também detectar fatores que possam comprometer o neurodesenvolvimento e a potencialidade intelectual. Essa grande variabilidade de influências sobre o crescimento do perímetro craniano pode ser realçada por muitos pesquisadores. No entanto, não nos permitiu, na presente pesquisa, estabelecer o motivo por que a criança com deficiência auditiva tem menor perímetro craniano.

No restante da avaliação neurológica do grupo controle, com produção escolar normal, foi encontrado hipotonia muscular de membros em 40 crianças e síndrome cerebelar apendicular em duas. Esses sinais isolados foram considerados como variação do normal, não sendo atribuidos significados patológicos.

No estudo do tono muscular em pré-escolares de São Paulo, Lefèvre e col. examinaram um conjunto de 200 crianças selecionadas rigorosamente em termos de normalidade, visando estabelecer um padrão de desenvolvimento neurológico 9. Entre essas crianças, foram encontrados dois casos de hipotonia muscular, que não foram considerados patológicos. Usando como critério de seleção a observação de fichas escolares, Rotta 14 avaliou 50 escolares com bom rendimento, entre 7 e 9 anos, encontrando hipotonia muscular em 11. Esta foi considerada um sinal neurológico isolado, dentro da variação normal.

Entre os deficientes auditivos, foi encontrado hipotonia muscular de membros em 26 crianças, sugerindo aumento no número de crianças hipotônicas com o aumento da idade cronológica.

A hipotonia muscular resulta de grande variedade de disfunções. Inevitavelmente segue a perda de retroalimentação proprioceptiva dos músculos, desde que a alça gama é essencial para a manutenção do tono muscular. Assim, é referido por Rapin 11 que o tono muscular está diminuido quando a disfunção afeta o neurônio motor inferior ou o músculo, sendo acompanhada de paresia e hipotrofia. A hipotonia que se origina no nervo periférico ou medula espinhal está, usualmente, associada a arreflexia tendinosa. A hipotonia de origem central pode estar associada a lesão cerebelar, a patologia nos gânglios da base ou, ainda, a lesão cerebral difusa, quando reflexos hiperativos e outros sinais de espasticidade podem acompanhar a hipotonia. Perda da função vestibular pode resultar em hipotonia muscular, especialmente em crianças. Porém, é desconhecida a prevalência da disfunção labiríntica em crianças hipotônicas e com audição normal.

Dessa maneira, nessas crianças hipotônicas, o ENT afastou alteraçōes em outros sistemas, porém, em próxima pesquisa, serão analisados sistematicamente os testes de função labirintica.

Concluindo, pela análise dos dados do ENT, os aspectos que demonstraram diferenças entre crianças controles e aquelas DA, foram o perímetro craniano e o tono muscular. No restante do exame não foram encontradas alterações capazes de distinguir os dois grupos, verificando-se pequena contribuição do ENT no sentido de caracterizar outros diagnósticos sindrômicos entre os DA. Porém, essa semiologia permitiu excluir outros comprometimentos sensoriais ou motores, confirmando a deficiência auditiva pura. 


\section{TEFERENCIAS}

1. Castagno LA, Carvalhal ML. Childhood severe-profound sensorineural deafness. Folha' Med (Br) 1985, 9:397-400.

2. Cowie VA. Microcephaly: a review of genetic implications in its causation. $J$ Ment Def Res 1987, 31:229-233.

3. Diament A. Contribuição para a padronização do exame neurológico de crianças normais no primeiro ano de viăa. Tese (Doutoramento). Faculdade de Medicina, Universidade de Săo Paulo. São Paulo, 1967.

4. Diament A, Rodrigues FW. Cephalic measures in normal pre-school 3 to 7 years of age. Arq Neuropsiquiatr 1976, 34:325-330.

5. Dolk $H$. The predictive value of microcephaly during the first years of life for mental retardation at seven years. Dev Med Child Neurol 1991, 33:974-983.

6. Gomes U, Rosado MLC, Moura-Ribeiro MVL, Ricco RG. Saúde perinatal em Ribeirão Preto, Brasil: distribuição do perímetro craniano em recém-nascidos. Arq Neuropsiquiatr 1989, $47: 392-396$.

7. Horak FB, Shumway-Cook A, Crowe TK, Black FO. Vestibular function and motor profeciency of children with impaired hearing or with learning disability and motor impairments. Dev Med Child Neurol 1988, 30:64-79.

8. Lefèvre AB. O exame neurológico da crianç. In Tolosa APM, Canelas HM (eds): Propedêutica neurológica: temas essenciais. São Paulo: Sarvier, 1971, p 421-440.

9. Lefèvre AB. Exame neurológico evolutivo do pré-escóar normal. São Paulo: Sarvier, 1977.

10. Rapin I. Hypoactive labyrinths and motor development. Clin Ped 1974, 13:922-937.

11. Rapin I. Children with brain dysfunction: neurology, cognition, language and behaviour. New York: Raven Press, 1982.

12. Rapin I. Children with hearing impairment. In Swaiman KF (ed): Pediatric neurology: principles and practice. Saint Louis: Mosby, 1989, Vol 2, p 895-908.

13. Rosado MLC, Barbieri MA, Bettiol H, Gomes VR, Moura-Ribeiro MVL. Crescimento craniano na criança. Arq Neuropsiquiatr 1989, 47:397-402.

14. Rotta NT. Avaliação neurológica Evolutiva, eletrencefalográfica e psicológica em crianças com rendimento escolar deficiente. Tese (Livre Docência) Faculdade de Medicina, Universidade Federal do Rio Grande do Sul. Porto Alegre, 1975.

15. Sells CJ. Microcephaly in ta normal school population. Pediatrics 1977, 59:262-265.

16. Towen BCL. Examination of the child with minor neurological dysfunction. London: Spastics International Medical Publications, 1979.

17. Weinberg WA, Dietz SG, Penick EC. Intelligence, reading achievement, physical size and social cliass. $J$ Pediatr 1974, 85:482-489.

18. Werver DD, Christian JC. Familial variation of head size and adjustment for parental head circunference. J Pediatr 1980, 96:990-996. 